\title{
Research Article \\ Explicit Form of the Inverse Matrices of Tribonacci Circulant Type Matrices
}

\author{
Li Liu and Zhaolin Jiang \\ Department of Mathematics, Linyi University, Linyi, Shandong 276000, China \\ Correspondence should be addressed to Zhaolin Jiang; jzh1208@sina.com
}

Received 9 July 2014; Accepted 25 August 2014

Academic Editor: Zidong Wang

Copyright ( 2015 L. Liu and Z. Jiang. This is an open access article distributed under the Creative Commons Attribution License, which permits unrestricted use, distribution, and reproduction in any medium, provided the original work is properly cited.

\begin{abstract}
It is a hot topic that circulant type matrices are applied to networks engineering. The determinants and inverses of Tribonacci circulant type matrices are discussed in the paper. Firstly, Tribonacci circulant type matrices are defined. In addition, we show the invertibility of Tribonacci circulant matrix and present the determinant and the inverse matrix based on constructing the transformation matrices. By utilizing the relation between left circulant, $g$-circulant matrices and circulant matrix, the invertibility of Tribonacci left circulant and Tribonacci $g$-circulant matrices is also discussed. Finally, the determinants and inverse matrices of these matrices are given, respectively.
\end{abstract}

\section{Introduction}

Circulant type matrices have important applications in various networks engineering. Exploiting the circulant structure of the channel matrices, Eghbali et al. [1] analysed the realistic near fast fading scenarios with circulant frequency selective channels. The optimum sampling in the one- and twodimensional (1D and 2D) wireless sensor networks (WSNs) with spatial temporally correlated data was studied with circulant matrices in [2]. The repeat space theory (RST) was extended to apply to carbon nanotubes and related molecular networks, where the corresponding matrices are pseudocirculant in [3]. Preconditioners obtained by circulant approximations of stochastic automata networks were considered in [4]. In [5], circulant mutation whose differential equations obtained neither are of repliator-type nor can they be transformed straightway into a linear equation was introduced into autocatalytic reaction networks. Jing and Jafarkhani [6] proposed distributed differential space-time codes that work for networks with any number of relays using circulant matrices. Wang and Cheng [7] studied the existence of doubly periodic travelling waves in cellular networks involving the discontinuous Heaviside step function by circulant matrix. Pais et al. [8] proved conditions for the existence of stable limit cycles arising from multiple distinct Hopf bifurcations of the dynamics in the case of circulant fitness matrices.

Circulant type matrices have been put on the firm basis with the work in $[9,10]$ and so on. Furthermore, the $g$ circulant matrices are focused on by many researchers; for the details please refer to [11-13] and the references therein.

Lately, some scholars gave the explicit determinant and inverse of the circulant and skew-circulant involving famous numbers. Jiang et al. [14] discussed the invertibility of circulant type matrices with the sum and product of Fibonacci and Lucas numbers and presented the determinants and the inverses of these matrices. Jiang et al. [15] considered circulant type matrices with the $k$-Fibonacci and $k$-Lucas numbers and presented the explicit determinant and inverse matrix by constructing the transformation matrices. Jiang and Hong [16] gave exact form determinants of the RSFPLR circulant matrices and the RSLPFL circulant matrices involving Perrin, Padovan, Tribonacci, and the generalized Lucas number by the inverse factorization of polynomial. Bozkurt and Tam gave determinants and inverses of circulant matrices with Jacobsthal and Jacobsthal-Lucas numbers in [17]. Cambini presented an explicit form of the inverse of a particular circulant matrix in [18]. Shen et al. considered circulant matrices 
with Fibonacci and Lucas numbers and presented their explicit determinants and inverses in [19].

The Tribonacci sequences are defined by the following recurrence relations [20-22], respectively:

$$
T_{n}=T_{n-1}+T_{n-2}+T_{n-3}
$$

where $T_{0}=0, T_{1}=1, T_{2}=1, n \geq 3$.

The first few values of the sequences are given by the following table:

$$
\begin{array}{c|cccccccccc}
n & 0 & 1 & 2 & 3 & 4 & 5 & 6 & 7 & 8 & \cdots \\
\hline T_{n} & 0 & 1 & 1 & 2 & 4 & 7 & 13 & 24 & 44 & \cdots
\end{array} .
$$

Let $t_{1}, t_{2}$, and $t_{3}$ be the roots of the characteristic equation $x^{3}-x^{2}-x-1=0$ and then we have

$$
\begin{gathered}
t_{1}+t_{2}+t_{3}=1, \\
t_{1} t_{2}+t_{1} t_{3}+t_{2} t_{3}=-1, \\
t_{1} t_{2} t_{3}=1 .
\end{gathered}
$$

Hence the Binet formulas of the sequences $\left\{T_{n}\right\}$ have the form

$$
T_{n}=b_{1} t_{1}^{n}+b_{2} t_{2}^{n}+b_{3} t_{3}^{n}
$$

where $b_{i}$ is the $i$ th root of the polynomial $44 y^{3}-2 y-1$ for $i=1,2,3$.

In this paper, we consider circulant type matrices, including the circulant and left circulant and $g$-circulant matrices. If we suppose $T_{n}$ is the $n$th Tribonacci number, then we define a Tribonacci circulant matrix which is an $n \times n$ matrix with the following form:

$$
\begin{aligned}
\operatorname{Circ} & \left(T_{1}, T_{2}, \cdots, T_{n}\right) \\
= & {\left[\begin{array}{cccc}
T_{1} & T_{2} & \cdots & T_{n} \\
T_{n} & T_{1} & \cdots & T_{n-1} \\
\vdots & \vdots & & \vdots \\
T_{2} & T_{3} & \cdots & T_{1}
\end{array}\right] . }
\end{aligned}
$$

Besides, a Tribonacci left circulant matrix is given by

$$
\begin{aligned}
& \operatorname{LCirc}\left(T_{1}, T_{2}, \cdots, T_{n}\right) \\
& =\left[\begin{array}{cccc}
T_{1} & T_{2} & \cdots & T_{n} \\
T_{2} & T_{3} & \cdots & T_{1} \\
\vdots & \vdots & & \vdots \\
T_{n} & T_{1} & \cdots & T_{n-1}
\end{array}\right],
\end{aligned}
$$

where each row is a cyclic shift of the row above to the left.

A Tribonacci $g$-circulant matrix is an $n \times n$ matrix with the following form:

$$
A_{g, n}=\left(\begin{array}{cccc}
T_{1} & T_{2} & \cdots & T_{n} \\
T_{n-g+1} & T_{n-g+2} & \cdots & T_{n-g} \\
T_{n-2 g+1} & T_{n-2 g+2} & \cdots & T_{n-2 g} \\
\vdots & \vdots & \ddots & \vdots \\
T_{g+1} & T_{g+2} & \cdots & T_{g}
\end{array}\right) \text {, }
$$

where $g$ is a nonnegative integer and each of the subscripts is understood to be reduced modulo $n$. The first row of $A_{g, n}$ is $\left(T_{1}, T_{2}, \ldots, T_{n}\right)$, and its $(j+1)$ th row is obtained by giving its $j$ th row a right circular shift by $g$ positions (equivalently, $g \bmod n$ positions). Note that $g=1$ or $g=n+1$ yields standard Tribonacci circulant matrix. If $g=n-1$, then we obtain Tribonacci left circulant matrix.

Lemma 1. The $n \times n$ tridiagonal matrix is given by

$$
A_{n}=\left(\begin{array}{ccccccc}
\tau_{2} & \tau_{1} & & & & & 0 \\
\tau_{3} & \tau_{2} & \tau_{1} & & & & \\
& \tau_{3} & \tau_{2} & \tau_{1} & & & \\
& & \ddots & \ddots & \ddots & & \\
& & & \tau_{3} & \tau_{2} & \tau_{1} & \\
& & & & \tau_{3} & \tau_{2} & \tau_{1} \\
0 & & & & & \tau_{3} & \tau_{2}
\end{array}\right)
$$

then

$\operatorname{det} A_{n}$

$$
=\left\{\begin{array}{l}
\frac{\left(\left(\tau_{2}+\sqrt{\tau_{2}^{2}-4 \tau_{1} \tau_{3}}\right) / 2\right)^{n+1}-\left(\left(\tau_{2}-\sqrt{\tau_{2}^{2}-4 \tau_{1} \tau_{3}}\right) / 2\right)^{n+1}}{\sqrt{\tau_{2}^{2}-4 \tau_{1} \tau_{3}}}, \\
(n+1)\left(\frac{\tau_{2}}{2}\right)^{n}, \quad \tau_{2}^{2}=4 \tau_{1} \tau_{3} .
\end{array}\right.
$$

Proof. $\operatorname{det} A_{n}=\tau_{2} \cdot \operatorname{det} A_{n-1}-\tau_{1} \tau_{3} \cdot \operatorname{det} A_{n-2} ;$ let $x+y=$ $\tau_{2}, x y=\tau_{1} \tau_{3}$ and then let $x, y$ be the roots of the equation $x^{2}-\tau_{2} x+\tau_{1} \tau_{3}=0$.

We have

$$
\begin{aligned}
\operatorname{det} A_{n}= & y^{n}+x y^{n-1}+\cdots+x^{n-1} y+x^{n} \\
& = \begin{cases}\frac{x^{n+1}-y^{n+1}}{x-y}, & x \neq y, \\
(n+1) x^{n}, & x=y,\end{cases}
\end{aligned}
$$

where $x=\left(\tau_{2}+\sqrt{\tau_{2}^{2}-4 \tau_{1} \tau_{3}}\right) / 2$ and $y=\left(\tau_{2}-\sqrt{\tau_{2}^{2}-4 \tau_{1} \tau_{3}}\right) / 2$.

Hence,

$\operatorname{det} A_{n}$

$$
=\left\{\begin{array}{l}
\frac{\left(\left(\tau_{2}+\sqrt{\tau_{2}^{2}-4 \tau_{1} \tau_{3}}\right) / 2\right)^{n+1}-\left(\left(\tau_{2}-\sqrt{\tau_{2}^{2}-4 \tau_{1} \tau_{3}}\right) / 2\right)^{n+1}}{\sqrt{\tau_{2}^{2}-4 \tau_{1} \tau_{3}},}, \\
(n+1)\left(\frac{\tau_{2}}{2}\right)^{n}, \quad \tau_{2}^{2}=4 \tau_{1} \tau_{3} .
\end{array}\right.
$$


Lemma 2. Let

$$
B_{n}=\left(\begin{array}{ccccccc}
a_{1} & a_{2} & a_{3} & \cdots & a_{n-2} & a_{n-1} & a_{n} \\
\tau_{2} & \tau_{1} & & & & & \\
\tau_{3} & \tau_{2} & \tau_{1} & & & & \\
& \tau_{3} & \tau_{2} & \tau_{1} & & & \\
& & \ddots & \ddots & \ddots & & \\
& & & \tau_{3} & \tau_{2} & \tau_{1} & \\
& & & & \tau_{3} & \tau_{2} & \tau_{1}
\end{array}\right)
$$

be an $n \times n$ matrix; one has

$$
\operatorname{det} B_{n}=\sum_{i=1}^{n}(-1)^{1+i} \tau_{1}^{n-i} a_{i} \cdot \operatorname{det} A_{i-1},
$$

where

$$
\begin{aligned}
& \operatorname{det} A_{i-1} \\
& =\left\{\begin{array}{l}
\frac{\left(\left(\tau_{2}+\sqrt{\tau_{2}^{2}-4 \tau_{1} \tau_{3}}\right) / 2\right)^{i}-\left(\left(\tau_{2}-\sqrt{\tau_{2}^{2}-4 \tau_{1} \tau_{3}}\right) / 2\right)^{i}}{\sqrt{\tau_{2}^{2}-4 \tau_{1} \tau_{3}},}, \\
i\left(\frac{\tau_{2}}{2}\right)^{i-1}, \quad \tau_{2}^{2}=4 \tau_{1} \tau_{3} .
\end{array}\right.
\end{aligned}
$$

Specifically, $\operatorname{det} A_{0}=1$.

Proof. Accoding to the last column determinant expansion and Lemma 1, we obtain

$$
\begin{aligned}
\operatorname{det} B_{n}= & \tau_{1} \cdot \operatorname{det} B_{n-1}+(-1)^{n+1} a_{n} \cdot \operatorname{det} A_{n-1} \\
= & \tau_{1}\left(\tau_{1} \cdot \operatorname{det} B_{n-2}+(-1)^{n} a_{n-1} \cdot \operatorname{det} A_{n-2}\right) \\
& +(-1)^{n+1} a_{n} \cdot \operatorname{det} A_{n-1} \\
= & \tau_{1}^{n-1} \cdot \operatorname{det} B_{1}+(-1)^{1+2} \tau_{1}^{n-2} a_{2} \cdot \operatorname{det} A_{1} \\
& +(-1)^{1+3} \tau_{1}^{n-3} a_{3} \cdot \operatorname{det} A_{2} \\
& +\cdots+(-1)^{1+n} a_{n} \cdot \operatorname{det} A_{n-1} \\
= & \sum_{i=1}^{n}(-1)^{1+i} \tau_{1}^{n-i} a_{i} \cdot \operatorname{det} A_{i-1} \cdot
\end{aligned}
$$

\section{Determinant and Inverse of Tibonacci Circulant Matrix}

In this section, let $D_{n}=\operatorname{Circ}\left(T_{1}, T_{2}, \ldots, T_{n}\right)$ be a Tribonacci circulant matrix. Firstly, we give the determinant of the matrix $D_{n}$. Afterwards, we discuss the invertibility of the matrix $D_{n}$, and we find the inverse of the matrix $D_{n}$.
Theorem 3. Let $D_{n}=\operatorname{Circ}\left(T_{1}, T_{2}, \ldots, T_{n}\right)$ be a Tribonacci circulant matrix; then we have

$$
\begin{aligned}
\operatorname{det} D_{n}= & {\left[T_{1}-T_{n}+\sum_{i=1}^{n-2} \Delta^{i}\left(T_{n-i+1}-T_{n-i}\right)\right] \delta_{1} } \\
& -\left[-T_{n}+\Delta\left(T_{1}-T_{n}-T_{n-1}\right)+\sum_{i=1}^{n-3} \Delta^{i+1} T_{n-i-2}\right] \delta_{2},
\end{aligned}
$$

where $T_{n}$ is the nth Tribonacci number, and

$$
\begin{aligned}
& \Delta=\frac{-b \pm \sqrt{b^{2}-4 a c}}{2 a} \\
& \delta_{1}=\left(T_{1}-T_{n}-T_{n-1}\right)\left(T_{1}-T_{n+1}\right)^{n-3} \\
& +\sum_{i=2}^{n-2}(-1)^{1+i}\left(T_{1}-T_{n+1}\right)^{n-i-2} T_{n-i-1} \cdot \operatorname{det} A_{i-1}, \\
& \delta_{2}=\sum_{i=1}^{n-2}(-1)^{1+i}\left(T_{1}-T_{n+1}\right)^{n-i-2}\left(T_{n-i+1}-T_{n-i}\right) \cdot \operatorname{det} A_{i-1}, \\
& \operatorname{det} A_{i-1} \\
& =\left\{\begin{array}{l}
\frac{\left(\left(b+\sqrt{b^{2}-4 a c}\right) / 2\right)^{i}-\left(\left(b-\sqrt{b^{2}-4 a c}\right) / 2\right)^{i}}{\sqrt{b^{2}-4 a c}}, \\
i\left(\frac{b}{2}\right)^{i-1}, \quad b^{2}=4 a c,
\end{array}\right. \\
& a=T_{1}-T_{n+1}, \\
& b=-T_{n}-T_{n-1} \text {, } \\
& c=-T_{n} \text {. }
\end{aligned}
$$

Proof. Obviously, det $D_{1}=1$ satisfies (16). In the case where $n>1$, let

$$
\Gamma_{1}=\left(\begin{array}{ccccccccc}
1 & & & & & & 0 \\
-1 & & & & & & 1 \\
-1 & & & & & 1 & -1 \\
-1 & & & & & 1 & -1 & -1 \\
0 & & & & 1 & -1 & -1 & -1 \\
\vdots & & & . & . & . & . & . & . \\
0 & & 1 & -1 & -1 & -1 & & \\
0 & 1 & -1 & -1 & -1 & & &
\end{array}\right),
$$

$$
\Pi_{1}=\left(\begin{array}{cccccc}
1 & 0 & 0 & \cdots & 0 & 0 \\
0 & \Delta^{n-2} & 0 & \cdots & 0 & 1 \\
0 & \Delta^{n-3} & 0 & \cdots & 1 & 0 \\
\vdots & \vdots & \vdots & . & \vdots & \vdots \\
0 & \Delta & 1 & \cdots & 0 & 0 \\
0 & 1 & 0 & \cdots & 0 & 0
\end{array}\right)
$$


be two $n \times n$ matrices and then we have

$$
\Gamma_{1} D_{n} \Pi_{1}=\left(\begin{array}{ccccccc}
T_{1} & p_{1} & T_{n-1} & T_{n-2} & \cdots & T_{3} & T_{2} \\
0 & p_{2} & \varphi_{3} & \varphi_{4} & \cdots & \varphi_{n-1} & \varphi_{n} \\
0 & p_{3} & \phi & T_{n-3} & \cdots & T_{2} & T_{1} \\
0 & 0 & b & a & & & \\
0 & 0 & c & b & a & & \\
& & & \ddots & \ddots & \ddots & \\
& & & & c & b & a
\end{array}\right)
$$

where

$$
\begin{aligned}
& p_{1}=\sum_{i=1}^{n-1} T_{i+1} \Delta^{n-(i+1)}, \\
& p_{2}=T_{1}-T_{n}+\sum_{i=1}^{n-2} \Delta^{i}\left(T_{n-i+1}-T_{n-i}\right) \\
& p_{3}=-T_{n}+\Delta\left(T_{1}-T_{n}-T_{n-1}\right)+\sum_{i=1}^{n-3} \Delta^{i+1} T_{n-i-2}, \\
& \varphi_{i}=T_{n+3-i}-T_{n+2-i}, \quad(i=3, \ldots, n), \\
& \phi=T_{1}-T_{n}-T_{n-1}, \quad \\
& \Delta=\frac{-b \pm \sqrt{b^{2}-4 a c}}{2 a}, \\
& a=T_{1}-T_{n+1}, \quad b=-T_{n}-T_{n-1}, \quad c=-T_{n} .
\end{aligned}
$$

We obtain

$$
\begin{aligned}
\operatorname{det} & \Gamma_{1} \operatorname{det} D_{n} \operatorname{det} \Pi_{1} \\
= & T_{1} \cdot\left(p_{2} \delta_{1}-p_{3} \delta_{2}\right) \\
= & {\left[T_{1}-T_{n}+\sum_{i=1}^{n-2} \Delta^{i}\left(T_{n-i+1}-T_{n-i}\right)\right] \delta_{1} } \\
& -\left[-T_{n}+\Delta\left(T_{1}-T_{n}-T_{n-1}\right)+\sum_{i=1}^{n-3} \Delta^{i+1} T_{n-i-2}\right] \delta_{2},
\end{aligned}
$$

where

$$
\begin{aligned}
\delta_{1}= & \left(T_{1}-T_{n}-T_{n-1}\right)\left(T_{1}-T_{n+1}\right)^{n-3} \\
& +\sum_{i=2}^{n-2}(-1)^{1+i}\left(T_{1}-T_{n+1}\right)^{n-i-2} \cdot T_{n-i-1} \cdot \operatorname{det} A_{i-1}, \\
\delta_{2}= & \sum_{i=1}^{n-2}(-1)^{1+i}\left(T_{1}-T_{n+1}\right)^{n-i-2}\left(T_{n-i+1}-T_{n-i}\right) \cdot \operatorname{det} A_{i-1} .
\end{aligned}
$$

Let

$$
\begin{aligned}
& \mathscr{B}_{n}=\left(\begin{array}{cccccc}
\phi & T_{n-3} & \cdots & T_{3} & T_{2} & T_{1} \\
b & a & & & & \\
c & b & a & & & \\
& \ddots & \ddots & \ddots & & \\
& & c & b & a & \\
& & & c & b & a
\end{array}\right), \\
& \mathscr{C}_{n}=\left(\begin{array}{cccccc}
\varphi_{3} & \varphi_{4} & \cdots & \varphi_{n-2} & \varphi_{n-1} & \varphi_{n} \\
b & a & & & & \\
c & b & a & & & \\
& \ddots & \ddots & \ddots & & \\
& & c & b & a &
\end{array}\right)
\end{aligned}
$$

be two $(n-2) \times(n-2)$ matrices, and $\delta_{1}=\operatorname{det} \mathscr{B}_{n}$, and $\delta_{2}=$ $\operatorname{det} \mathscr{C}_{n}$.

Aoccording to Lemma 2, thus

$$
\begin{aligned}
\delta_{1}= & \left(T_{1}-T_{n}-T_{n-1}\right)\left(T_{1}-T_{n+1}\right)^{n-3} \\
& +\sum_{i=2}^{n-2}(-1)^{1+i}\left(T_{1}-T_{n+1}\right)^{n-i-2} \cdot T_{n-i-1} \cdot \operatorname{det} A_{i-1}, \\
\delta_{2}= & \sum_{i=1}^{n-2}(-1)^{1+i}\left(T_{1}-T_{n+1}\right)^{n-i-2}\left(T_{n-i+1}-T_{n-i}\right) \cdot \operatorname{det} A_{i-1} .
\end{aligned}
$$

While

$$
\operatorname{det} \Gamma_{1}=\operatorname{det} \Pi_{1}=(-1)^{(n-1)(n-2) / 2} \text {, }
$$

we have

$$
\begin{aligned}
\operatorname{det} D_{n}= & {\left[T_{1}-T_{n}+\sum_{i=1}^{n-2} \Delta^{i}\left(T_{n-i+1}-T_{n-i}\right)\right] \delta_{1} } \\
& -\left[-T_{n}+\Delta\left(T_{1}-T_{n}-T_{n-1}\right)+\sum_{i=1}^{n-3} \Delta^{i+1} T_{n-i-2}\right] \delta_{2} .
\end{aligned}
$$

Theorem 4. Let $D_{n}=\operatorname{Circ}\left(T_{1}, T_{2}, \ldots, T_{n}\right)$ be a Tribonacci circulant matrix; if $n \neq 2$ and $n \neq 2 k \pi\left(\arctan \left( \pm \sqrt{4 a c-b^{2}}\right.\right.$ / $-b))^{-1}(k=1,2, \ldots, n-1)$, then $D_{n}$ is an invertible matrix.

Proof. When $n=2$ in Theorem 3, then we have $\operatorname{det} D_{2}=0$. Hence, $D_{2}$ is not invertible.

In the case where $n>2$, since $T_{n}=b_{1} t_{1}^{n}+b_{2} t_{2}^{n}+b_{3} t_{3}^{n}$, where $b_{i}$ is the $i$ th root of the polynomial $44 y^{3}-2 y-1$, we have

$$
\begin{aligned}
f\left(\omega^{k}\right) & =\sum_{j=1}^{n} T_{j}\left(\omega^{k}\right)^{j-1} \\
& =\sum_{j=1}^{n}\left(b_{1} t_{1}^{j}+b_{2} t_{2}^{j}+b_{3} t_{3}^{j}\right)\left(\omega^{k}\right)^{j-1}
\end{aligned}
$$




$$
\begin{aligned}
& =\frac{b_{1} t_{1}\left(1-t_{1}^{n}\right)}{1-t_{1} \omega^{k}}+\frac{b_{2} t_{2}\left(1-t_{2}^{n}\right)}{1-t_{2} \omega^{k}}+\frac{b_{3} t_{3}\left(1-t_{3}^{n}\right)}{1-t_{3} \omega^{k}} \\
& =\frac{1-T_{n+1}+\left(-T_{n}-T_{n-1}\right) \omega^{k}-T_{n} \omega^{2 k}}{1-\omega^{k}-\omega^{2 k}-\omega^{3 k}} \\
& \quad(k=1,2, \ldots, n-1) .
\end{aligned}
$$

If there exists $\omega^{l}(l=1,2, \ldots, n-1)$ such that $f\left(\omega^{l}\right)=$ 0 , we obtain $1-T_{n+1}+\left(-T_{n}-T_{n-1}\right) \omega^{k}-T_{n} \omega^{2 k}=0$ for $1-\omega^{k}-\omega^{2 k}-\omega^{3 k} \neq 0$. Thus, $\omega^{l}=\left(T_{n}+T_{n-1} \pm\right.$ $\left.\sqrt{\left(-T_{n}-T_{n-1}\right)^{2}+4 T_{n}\left(T_{1}-T_{n+1}\right)}\right) /-2 T_{n}$.

Let $a=T_{1}-T_{n+1}, b=-T_{n}-T_{n-1}$, and $c=-T_{n}$; if $b^{2}-4 a c \geq$ 0 , we have $\omega^{l}$ is a real number.

While

$$
\omega^{l}=\exp \left(\frac{2 l \pi i}{n}\right)=\cos \left(\frac{2 l \pi}{n}\right)+i \sin \left(\frac{2 l \pi}{n}\right)
$$

$\sin (2 l \pi / n)=0$. We have $\omega^{l}=-1$ for $0<(2 l \pi / n)<2 \pi$, but $1-\omega^{k}-\omega^{2 k}-\omega^{3 k}=0$. We obtain $f\left(\omega^{k}\right) \neq 0$ for any $\omega^{k}(k=1,2, \ldots, n-1)$, while $f(1)=\sum_{j=1}^{n} T_{j}=-(1 / 2)(1-$ $\left.T_{n}-T_{n+2}\right) \neq 0$.

If $b^{2}-4 a c<0, \omega^{l}$ is an imaginary number.

If

$$
\begin{aligned}
& \cos \left(\frac{2 l \pi}{n}\right)=\frac{T_{n}+T_{n-1}}{-2 T_{n}} \\
& \sin \left(\frac{2 l \pi}{n}\right)=\frac{\sqrt{-4 T_{n}\left(T_{1}-T_{n+1}\right)-\left(-T_{n}-T_{n-1}\right)^{2}}}{-2 T_{n}}
\end{aligned}
$$

or

$$
\begin{aligned}
& \cos \left(\frac{2 l \pi}{n}\right)=\frac{T_{n}+T_{n-1}}{-2 T_{n}}, \\
& \sin \left(\frac{2 l \pi}{n}\right)=-\frac{\sqrt{-4 T_{n}\left(T_{1}-T_{n+1}\right)-\left(-T_{n}-T_{n-1}\right)^{2}}}{-2 T_{n}},
\end{aligned}
$$

we obtain $n=2 k \pi\left(\arctan \left( \pm \sqrt{4 a c-b^{2}} /-b\right)\right)^{-1}$, such that $f\left(\omega^{l}\right)=0$. If $1-\omega^{k}-\omega^{2 k}-\omega^{3 k}=0$, we have $\omega^{k}=-1$ and if $n$ is an even number, then $f\left(\omega^{k}\right)=\sum_{j=1}^{n} T_{j}\left(\omega^{k}\right)^{j-1}=$ $T_{1}-T_{2}+\cdots-T_{n}<0$. By Lemma 1 in [15], the proof is completed.
Lemma 5. Let $\Phi=\left(\begin{array}{cc}a & V \\ U & A\end{array}\right)$ be an $n \times n$ matrix; then

$$
\Phi^{-1}=\left(\begin{array}{cc}
\frac{1}{\ell} & -\frac{1}{\ell} V A^{-1} \\
-\frac{1}{\ell} A^{-1} U & A^{-1}+\frac{1}{\ell} A^{-1} U V A^{-1}
\end{array}\right),
$$

where $\ell=a-V A^{-1} U, V$ is a row vector, and $U$ is a column vector.

Proof. Consider

$$
\begin{aligned}
& \left(\begin{array}{cc}
a & V \\
U & A
\end{array}\right) \cdot\left(\begin{array}{cc}
\frac{1}{\ell} & -\frac{1}{\ell} V A^{-1} \\
-\frac{1}{\ell} A^{-1} U & A^{-1}+\frac{1}{\ell} A^{-1} U V A^{-1}
\end{array}\right) \\
& =\left(\begin{array}{cc}
1 & 0 \\
0 & I_{n-1}
\end{array}\right)=I_{n}, \\
& \left(\begin{array}{cc}
\frac{1}{\ell} & -\frac{1}{\ell} V A^{-1} \\
-\frac{1}{\ell} A^{-1} U & A^{-1}+\frac{1}{\ell} A^{-1} U V A^{-1}
\end{array}\right) \cdot\left(\begin{array}{cc}
a & V \\
U & A
\end{array}\right) \\
& =\left(\begin{array}{cc}
1 & 0 \\
0 & I_{n-1}
\end{array}\right)=I_{n} .
\end{aligned}
$$

Lemma 6. Let the matrix $\mathscr{G}=\left[g_{i, j}\right]_{i, j=1}^{n-3}$ be of the form

$$
g_{i, j}= \begin{cases}T_{1}-T_{n+1}, & i=j, \\ -T_{n}-T_{n-1}, & i=j+1, \\ -T_{n}, & i=j+2, \\ 0, & \text { otherwise }\end{cases}
$$

then the inverse $\mathscr{G}^{-1}=\left[g_{i, j}^{\prime}\right]_{i, j=1}^{n-3}$ of the matrix $\mathscr{G}$ is equal to

$$
g_{i, j}^{\prime}= \begin{cases}\frac{1}{T_{1}-T_{n+1}}\left(\frac{\beta^{i-j+1}-\alpha^{i-j+1}}{\beta-\alpha}\right), & i \geq j, \\ 0, & i<j,\end{cases}
$$

where

$$
\begin{gathered}
\alpha=-\frac{\left(-T_{n}-T_{n-1}\right)+\sqrt{\left(-T_{n}-T_{n-1}\right)^{2}+4 T_{n}\left(T_{1}-T_{n+1}\right)}}{2\left(T_{1}-T_{n+1}\right)}, \\
\beta=-\frac{\left(-T_{n}-T_{n-1}\right)-\sqrt{\left(-T_{n}-T_{n-1}\right)^{2}+4 T_{n}\left(T_{1}-T_{n+1}\right)}}{2\left(T_{1}-T_{n+1}\right)} .
\end{gathered}
$$

Proof. Let $c_{i, j}=\sum_{k=1}^{n-3} g_{i, k} g_{k, j}^{\prime}$. Obviously, $c_{i, j}=0$ for $i<j$. In the case where $i=j$, we obtain

$$
c_{i, i}=g_{i, i} g_{i, i}^{\prime}=\left(T_{1}-T_{n+1}\right) \cdot \frac{1}{T_{1}-T_{n+1}}=1
$$


For $i \geq j+1$, we obtain

$$
\begin{aligned}
c_{i, j}= & \sum_{k=1}^{n-3} g_{i, k} g_{k, j}^{\prime} \\
= & g_{i, i-2} g_{i-2, j}^{\prime}+g_{i, i-1} g_{i-1, j}^{\prime}+g_{i, i} g_{i, j}^{\prime} \\
= & -T_{n} \cdot \frac{1}{T_{1}-T_{n+1}}\left(\frac{\beta^{i-j-1}-\alpha^{i-j-1}}{\beta-\alpha}\right) \\
& +\left(-T_{n}-T_{n-1}\right) \cdot \frac{1}{T_{1}-T_{n+1}}\left(\frac{\beta^{i-j}-\alpha^{i-j}}{\beta-\alpha}\right) \\
& +\left(T_{1}-T_{n+1}\right) \cdot \frac{1}{T_{1}-T_{n+1}}\left(\frac{\beta^{i-j+1}-\alpha^{i-j+1}}{\beta-\alpha}\right) \\
= & 0 .
\end{aligned}
$$

Hence, we verify $\mathscr{G} \mathscr{G}^{-1}=I_{n-3}$, where $I_{n-3}$ is $(n-3) \times(n-3)$ identity matrix. Similarly, we can verify $\mathscr{G}^{-1} \mathscr{G}=I_{n-3}$. Thus, the proof is completed.

Theorem 7. Let $D_{n}=\operatorname{Circ}\left(T_{1}, T_{2}, \ldots, T_{n}\right)$ be an invertible Tribonacci circulant matrix; then one has

$$
\begin{aligned}
D_{n}^{-1}=\operatorname{Circ}\left(x_{2}^{\prime}+\right. & \left(-1-\frac{p_{3}}{p_{2}}\right) x_{3}^{\prime}-x_{4}^{\prime}-x_{5}^{\prime}, \\
& -x_{2}^{\prime}+\left(-1+\frac{p_{3}}{p_{2}}\right) x_{3}^{\prime}-x_{4}^{\prime}, x_{n}^{\prime}, x_{n-1}^{\prime}-x_{n}^{\prime}, \\
& \left.x_{n-2}^{\prime}-x_{n-1}^{\prime}-x_{n}^{\prime}, \ldots, x_{3}^{\prime}-x_{4}^{\prime}-x_{5}^{\prime}-x_{6}^{\prime}\right),
\end{aligned}
$$

where

$$
\begin{aligned}
& p_{2}=T_{1}-T_{n}+\sum_{i=1}^{n-2} \Delta^{i}\left(T_{n-i+1}-T_{n-i}\right), \\
& p_{3}=-T_{n}+\Delta\left(T_{1}-T_{n}-T_{n-1}\right)+\sum_{i=1}^{n-3} \Delta^{i+1} T_{n-i-2}, \\
& x_{1}^{\prime}=0 \\
& x_{2}^{\prime}=\frac{1}{p_{2}}, \\
& x_{3}^{\prime}=\frac{\gamma_{3}}{\ell}+\frac{\left(T_{1}-T_{n+1}\right) \sum_{i=2}^{n-2} g_{i 1} \gamma_{i+2}}{\ell}, \\
& x_{4}^{\prime}=-\frac{\gamma_{3} \sum_{i=2}^{n-3} g_{i 1} \rho_{i+3}}{\ell}+\sum_{i=1}^{n-3} g_{i 1} \gamma_{i+3}
\end{aligned}
$$

$$
-\frac{\left(T_{1}-T_{n+1}\right) \sum_{i=1}^{n-3} g_{i 1} \rho_{i+3} \sum_{i=2}^{n-2} g_{i 1} \gamma_{i+2}}{\ell}
$$

$$
\begin{aligned}
x_{k}^{\prime}= & -\frac{\gamma_{3} \sum_{i=2}^{n-k+1} g_{i 1} \rho_{i+k-1}}{\ell}+\sum_{i=1}^{n-k+1} g_{i 1} \gamma_{i+k-1} \\
& -\frac{\left(T_{1}-T_{n+1}\right) \sum_{i=1}^{n-k+1} g_{i 1} \rho_{i+k-1} \sum_{i=2}^{n-2} g_{i 1} \gamma_{i+2}}{\ell},
\end{aligned}
$$

Proof. Let

$$
\Gamma_{2}=\left(\begin{array}{ccccc}
1 & 0 & 0 & \cdots & 0 \\
0 & 1 & & & \\
0 & -\frac{p_{3}}{p_{2}} & 1 & 0 & 0 \\
\cdots & \cdots & \cdots & \ddots & \cdots \\
0 & 0 & 0 & \cdots & 1
\end{array}\right)
$$

thus

$$
\Gamma_{2} \Gamma_{1} D_{n} \Pi_{1}=\left(\begin{array}{ccccccc}
T_{1} & p_{1} & T_{n-1} & T_{n-2} & \cdots & T_{3} & T_{2} \\
0 & p_{2} & \varphi_{3} & \varphi_{4} & \cdots & \varphi_{n-1} & \varphi_{n} \\
0 & 0 & \rho_{3} & \rho_{4} & \cdots & \rho_{n-1} & \rho_{n} \\
0 & 0 & b & a & & & \\
0 & 0 & c & b & a & & \\
& & & \ddots & \ddots & \ddots & \\
& & & & c & b & a
\end{array}\right),
$$

where

$$
\begin{gathered}
\rho_{3}=T_{1}-T_{n}-T_{n-1}-\frac{p_{3}}{p_{2}}\left(T_{n}-T_{n-1}\right), \\
\rho_{i}=T_{n-i+1}-\frac{p_{3}}{p_{2}}\left(T_{n-i+3}-T_{n-i+2}\right) \\
(i=4,5, \cdots, n), \\
\varphi_{i}=T_{n+3-i}-T_{n+2-i}, \quad(i=3, \ldots, n) .
\end{gathered}
$$

According to Lemma 5, let

$$
F=\left(\begin{array}{ll}
\rho_{3} & V \\
U & \mathscr{G}
\end{array}\right)
$$

be an $(n-2) \times(n-2)$ matrix and we obtain

$$
F^{-1}=\left(\begin{array}{cc}
\frac{1}{\ell} & -\frac{V \mathscr{G}^{-1}}{\ell} \\
-\frac{\mathscr{G}^{-1} U}{\ell} & \mathscr{G}^{-1}+\frac{U V \mathscr{G}^{-1}}{\ell}
\end{array}\right)
$$


where

$$
\begin{aligned}
\rho_{3} & =T_{1}-T_{n}-T_{n-1}-\frac{p_{3}}{p_{2}}\left(T_{n}-T_{n-1}\right), \\
U & =\left(-T_{n}-T_{n-1},-T_{n}, 0, \ldots, 0\right)^{T}, \\
V & =\left(\rho_{4}, \rho_{5}, \ldots, \rho_{n}\right), \\
\ell & =\rho_{3}-b \sum_{i=1}^{n-3} g_{i 1} \rho_{i+3}-c \sum_{i=1}^{n-4} \rho_{i+4} .
\end{aligned}
$$

Let

$$
\Pi_{2}=\left(\begin{array}{ccccc}
1 & -p_{1} & -T_{n-1}+\frac{p_{1} \varphi_{3}}{p_{2}} & \cdots & -T_{2}+\frac{p_{1} \varphi_{n}}{p_{2}} \\
0 & 1 & \frac{-\varphi_{3}}{p_{2}} & \cdots & \frac{-\varphi_{n}}{p_{2}} \\
0 & 0 & 1 & \cdots & 0 \\
\vdots & \vdots & \vdots & \ddots & \vdots \\
0 & 0 & 0 & \cdots & 1
\end{array}\right)
$$

where

$$
\begin{aligned}
& p_{1}=\sum_{i=1}^{n-1} T_{i+1} \Delta^{n-(i+1)}, \\
& p_{2}=T_{1}-T_{n}+\sum_{i=1}^{n-2} \Delta^{i}\left(T_{n-i+1}-T_{n-i}\right), \\
& \varphi_{i}=T_{n+3-i}-T_{n+2-i}, \quad(i=3, \ldots, n), \\
& \Delta=\frac{-b \pm \sqrt{b^{2}-4 a c}}{2 a}, \\
& a=T_{1}-T_{n+1}, \quad b=-T_{n}-T_{n-1}, \quad c=-T_{n} .
\end{aligned}
$$

We have

$$
\Gamma_{2} \Gamma_{1} D_{n} \Pi_{1} \Pi_{2}=\mathscr{D}_{1} \oplus F,
$$

where $\mathscr{D}_{1}=\operatorname{diag}\left(T_{1}, p_{2}\right)$ is a diagonal matrix and $\mathscr{D}_{1} \oplus F$ is the direct sum of $\mathscr{D}_{1}$ and $F$. If we denote $\Gamma=\Gamma_{2} \Gamma_{1}$ and $\Pi=\Pi_{1} \Pi_{2}$, we obtain

$$
D_{n}^{-1}=\Pi\left(\mathscr{D}_{1}^{-1} \oplus F^{-1}\right) \Gamma .
$$

Since the last row elements of the matrix $\Pi$ are $0,1,\left(T_{n-1}-\right.$ $\left.T_{n}\right) / p_{2},\left(T_{n-2}-T_{n-1}\right) / p_{2}, \ldots,\left(T_{3}-T_{4}\right) / p_{2},\left(T_{2}-T_{3}\right) / p_{2}$, then the elements of last row of $\Pi\left(\mathscr{D}_{1}^{-1} \oplus F^{-1}\right)$ are given by the following equations:

$$
\begin{aligned}
x_{1}^{\prime}= & 0, \\
x_{2}^{\prime}= & \frac{1}{p_{2}}, \\
x_{3}^{\prime}= & \frac{\gamma_{3}}{\ell}+\frac{\left(T_{1}-T_{n+1}\right) \sum_{i=2}^{n-2} g_{i 1} \gamma_{i+2}}{\ell}, \\
x_{4}^{\prime}= & -\frac{\gamma_{3} \sum_{i=2}^{n-3} g_{i 1} \rho_{i+3}}{\ell}+\sum_{i=1}^{n-3} g_{i 1} \gamma_{i+3} \\
& -\frac{\left(T_{1}-T_{n+1}\right) \sum_{i=1}^{n-3} g_{i 1} \rho_{i+3} \sum_{i=2}^{n-2} g_{i 1} \gamma_{i+2}}{\ell},
\end{aligned}
$$

$$
\begin{array}{r}
x_{k}^{\prime}=-\frac{\gamma_{3} \sum_{i=2}^{n-k+1} g_{i 1} \rho_{i+k-1}}{\ell}+\sum_{i=1}^{n-k+1} g_{i 1} \gamma_{i+k-1} \\
-\frac{\left(T_{1}-T_{n+1}\right) \sum_{i=1}^{n-k+1} g_{i 1} \rho_{i+k-1} \sum_{i=2}^{n-2} g_{i 1} \gamma_{i+2}}{\ell}, \\
(k \geq 4),
\end{array}
$$

where

$$
\begin{gathered}
\gamma_{i}=\frac{T_{n-i+2}-T_{n-i+3}}{p_{2}}, \quad(i=3,4 \ldots, n), \\
\rho_{3}=T_{1}-T_{n}-T_{n-1}-\frac{p_{3}}{p_{2}}\left(T_{n}-T_{n-1}\right), \\
\rho_{i}=T_{n-i+1}-\frac{p_{3}}{p_{2}}\left(T_{n-i+3}-T_{n-i+2}\right) \\
(i=4,5, \ldots, n) .
\end{gathered}
$$

By Lemma 6, if $D_{n}^{-1}=\operatorname{Circ}\left(x_{1}, x_{2}, \ldots, x_{n}\right)$, then its last row elements are given by the following equations:

$$
\begin{aligned}
x_{2}= & -x_{2}^{\prime}+\left(-1+\frac{p_{3}}{p_{2}}\right) x_{3}^{\prime}-x_{4}^{\prime}, \\
x_{3}= & x_{n}^{\prime}, \\
x_{4}= & x_{n-1}^{\prime}-x_{n}^{\prime}, \\
x_{5}= & x_{n-2}^{\prime}-x_{n-1}^{\prime}-x_{n}^{\prime}, \\
& \vdots \\
x_{k}= & x_{n-k+3}^{\prime}-x_{n-k+4}^{\prime}-x_{n-k+5}^{\prime}-x_{n-k+6}^{\prime} \\
x_{1}= & x_{2}^{\prime}+\left(-1-\frac{p_{3}}{p_{2}}\right) x_{3}^{\prime}-x_{4}^{\prime}-x_{5}^{\prime} .
\end{aligned}
$$

Hence, the proof is compeleted. 


\section{Determinant and Inverse of Tibonacci Left Circulant Matrix}

In this section, let $D_{n}^{\prime}=\operatorname{LC} \operatorname{Circ}\left(T_{1}, T_{2}, \ldots, T_{n}\right)$ be a Tibonacci left circulant matrix. By using the obtained conclusions, we give a determinant formula for the matrix $D_{n}^{\prime}$. Afterwards, we discuss the invertibility of the matrix $D_{n}^{\prime}$. The inverse of the matrix $D_{n}^{\prime}$ is also presented. According to Lemma 2 in [15] and Theorems 3, 4, and 7, we can obtain the following theorems.

Theorem 8. Let $D_{n}^{\prime}=\operatorname{LCirc}\left(T_{1}, T_{2}, \ldots, T_{n}\right)$ be a Tribonacci left circulant matrix; then one has

$$
\begin{aligned}
\operatorname{det} D_{n}^{\prime}=(-1)^{(n-1)(n-2) / 2} & \\
\times & {\left[\left[T_{1}-T_{n}+\sum_{i=1}^{n-2} \Delta^{i}\left(T_{n-i+1}-T_{n-i}\right)\right] \delta_{1}\right.} \\
& -\left[-T_{n}+\Delta\left(T_{1}-T_{n}-T_{n-1}\right)\right. \\
& \left.\left.+\sum_{i=1}^{n-3} \Delta^{i+1} T_{n-i-2}\right] \delta_{2}\right]
\end{aligned}
$$

where $T_{n}$ is the nth Tribonacci number.

Theorem 9. Let $D_{n}^{\prime}=\operatorname{LCirc}\left(T_{1}, T_{2}, \ldots, T_{n}\right)$ be a Tribonacci left circulant matrix. If $n \neq 2$ and $n \neq$ $2 k \pi\left(\arctan \left( \pm \sqrt{4 a c-b^{2}} /-b\right)\right)^{-1}(k=1,2, \ldots, n-1)$, then $D_{n}^{\prime}$ is an invertible matrix.

Theorem 10. Let $D_{n}^{\prime}=\operatorname{LCirc}\left(T_{1}, T_{2}, \ldots, T_{n}\right)$ be a Tribonacci left circulant matrix. If $D_{n}$ is an invertible matrix, then we have

$$
\begin{aligned}
D_{n}^{\prime-1}=\operatorname{Circ}\left(x_{2}^{\prime}+\right. & \left(-1-\frac{p_{3}}{p_{2}}\right) x_{3}^{\prime}-x_{4}^{\prime}-x_{5}^{\prime}, \\
& x_{3}^{\prime}-x_{4}^{\prime}-x_{5}^{\prime}-x_{6}^{\prime}, \ldots, x_{n-2}^{\prime}-x_{n-1}^{\prime}-x_{n}^{\prime}, \\
& \left.x_{n-1}^{\prime}-x_{n}^{\prime}, x_{n}^{\prime},-x_{2}^{\prime}+\left(-1+\frac{p_{3}}{p_{2}}\right) x_{3}^{\prime}-x_{4}^{\prime}\right),
\end{aligned}
$$

where

$$
\begin{aligned}
& x_{1}^{\prime}=0, \\
& x_{2}^{\prime}=\frac{1}{p_{2}}, \\
& x_{3}^{\prime}=\frac{\gamma_{3}}{\ell}+\frac{\left(T_{1}-T_{n+1}\right) \sum_{i=2}^{n-2} g_{i 1} \gamma_{i+2}}{\ell}, \\
& x_{4}^{\prime}=-\frac{\gamma_{3} \sum_{i=2}^{n-3} g_{i 1} \rho_{i+3}}{\ell}+\sum_{i=1}^{n-3} g_{i 1} \gamma_{i+3}
\end{aligned}
$$

$$
-\frac{\left(T_{1}-T_{n+1}\right) \sum_{i=1}^{n-3} g_{i 1} \rho_{i+3} \sum_{i=2}^{n-2} g_{i 1} \gamma_{i+2}}{\ell}
$$

$$
\begin{aligned}
x_{k}^{\prime}= & -\frac{\gamma_{3} \sum_{i=2}^{n-k+1} g_{i 1} \rho_{i+k-1}}{\ell}+\sum_{i=1}^{n-k+1} g_{i 1} \gamma_{i+k-1} \\
& -\frac{\left(T_{1}-T_{n+1}\right) \sum_{i=1}^{n-k+1} g_{i 1} \rho_{i+k-1} \sum_{i=2}^{n-2} g_{i 1} \gamma_{i+2}}{\ell},
\end{aligned}
$$

$(k \geq 4)$.

\section{Determinant and Inverse of Tibonacci $g$-Circulant Matrix}

In this section, let $D_{g, n}=g-\operatorname{Circ}\left(T_{1}, T_{2}, \ldots, T_{n}\right)$ be a Tibonacci $g$-circulant matrix. By using the obtained conclusions, we give a determinant formula for the matrix $D_{g, n}$. Afterwards, we discuss the invertibility of the matrix $D_{g, n}$. The inverse of the matrix $D_{g, n}$ is also presented. From Lemmas 3 and 4 in [15] and Theorems 3, 4, and 7, we deduce the following results.

Theorem 11. Let $D_{g, n}=g-\operatorname{Circ}\left(T_{1}, T_{2}, \ldots, T_{n}\right)$ be a Tribonacci $g$-circulant matrix and $(n, g)=1$; then one has

$$
\begin{gathered}
\operatorname{det} D_{g, n}=\operatorname{det} \mathbb{Q}_{g} \cdot[ \\
-\left[T_{1}-T_{n}+\sum_{i=1}^{n-2} \Delta^{i}\left(T_{n-i+1}-T_{n-i}\right)\right] \delta_{1} \\
-\left[T_{n} \Delta\left(T_{1}-T_{n}-T_{n-1}\right)\right. \\
\left.\left.+\sum_{i=1}^{n-3} \Delta^{i+1} T_{n-i-2}\right] \delta_{2}\right],
\end{gathered}
$$

where $T_{n}$ is the nth Tribonacci number.

Theorem 12. Let $D_{g, n}=g-\operatorname{Circ}\left(T_{1}, T_{2}, \ldots, T_{n}\right)$ be a Tribonacci g-circulant matrix and $(n, g)=1$. If $n \neq 2$ and $n \neq 2 k \pi\left(\arctan \left( \pm \sqrt{4 a c-b^{2}} /-b\right)\right)^{-1}(k=1,2, \ldots, n-1)$, then $D_{g, n}$ is an invertible matrix.

Theorem 13. Let $D_{g, n}=g$-Circ $\left(T_{1}, T_{2}, \ldots, T_{n}\right)$ be a Tribonacci $g$-circulant matrix and $(n, g)=1$. If $D_{n}$ is an invertible matrix, then one has

$$
\begin{aligned}
D_{g, n}^{-1}= & {\left[\operatorname { C i r c } \left(x_{2}^{\prime}+\left(-1-\frac{p_{3}}{p_{2}}\right) x_{3}^{\prime}-x_{4}^{\prime}-x_{5}^{\prime},\right.\right.} \\
& -x_{2}^{\prime}+\left(-1+\frac{p_{3}}{p_{2}}\right) x_{3}^{\prime}-x_{4}^{\prime}, x_{n}^{\prime}, x_{n-1}^{\prime}-x_{n}^{\prime}, x_{n-2}^{\prime} \\
& \left.\left.-x_{n-1}^{\prime}-x_{n}^{\prime}, \ldots, x_{3}^{\prime}-x_{4}^{\prime}-x_{5}^{\prime}-x_{6}^{\prime}\right)\right] \mathbb{Q}_{g}^{T},
\end{aligned}
$$


where

$$
\begin{aligned}
x_{1}^{\prime}= & 0, \\
x_{2}^{\prime}= & \frac{1}{p_{2}}, \\
x_{3}^{\prime}= & \frac{\gamma_{3}}{\ell}+\frac{\left(T_{1}-T_{n+1}\right) \sum_{i=2}^{n-2} g_{i 1} \gamma_{i+2}}{\ell}, \\
x_{4}^{\prime}= & -\frac{\gamma_{3} \sum_{i=2}^{n-3} g_{i 1} \rho_{i+3}}{\ell}+\sum_{i=1}^{n-3} g_{i 1} \gamma_{i+3} \\
& -\frac{\left(T_{1}-T_{n+1}\right) \sum_{i=1}^{n-3} g_{i 1} \rho_{i+3} \sum_{i=2}^{n-2} g_{i 1} \gamma_{i+2}}{\ell}, \\
& \vdots \\
x_{k}^{\prime}= & -\frac{\gamma_{3} \sum_{i=2}^{n-k+1} g_{i 1} \rho_{i+k-1}}{\ell}+\sum_{i=1}^{n-k+1} g_{i 1} \gamma_{i+k-1} \\
& -\frac{\left(T_{1}-T_{n+1}\right) \sum_{i=1}^{n-k+1} g_{i 1} \rho_{i+k-1} \sum_{i=2}^{n-2} g_{i 1} \gamma_{i+2}}{\ell},
\end{aligned}
$$

\section{Conclusion}

The related problem of Tribonacci circualnt type matrices is studied in this paper. We not only discuss nonsingularity of Tibonacci circulant type matrices, but also give the explicit determinant and inverse of Tribonacci circulant matrix, Tribonacci left circualant matrix, and Tribonacci $g$-circualant matrix. Furthermore, according to Theorem 11 in [23] and the result in Theorem 3 in the paper, identities can be easily obtained:

$$
\begin{aligned}
& \frac{\left(1-T_{n+1}\right)^{n}-\left(c_{1}^{n}+d_{1}^{n}\right)+\left(-T_{n}\right)^{n}}{\mathbb{L}_{-n}-\mathbb{L}_{n}} \\
& =\left[T_{1}-T_{n}+\sum_{i=1}^{n-2} \Delta^{i}\left(T_{n-i+1}-T_{n-i}\right)\right] \delta_{1} \\
& \quad-\left[-T_{n}+\Delta\left(T_{1}-T_{n}-T_{n-1}\right)+\sum_{i=1}^{n-3} \Delta^{i+1} T_{n-i-2}\right] \delta_{2},
\end{aligned}
$$

where $T_{n}$ is the $n$th Tribonacci number, $\mathbb{L}_{n}$ is the $n$th generalized Lucas number, and

$$
\begin{aligned}
& c_{1}=\frac{\left(T_{n+2}-T_{n+1}\right)+\mu_{1}}{2}, \\
& d_{1}=\frac{\left(T_{n+2}-T_{n+1}\right)-\mu_{1}}{2}, \\
& \mu_{1}=\sqrt{\left(T_{n+2}-T_{n+1}\right)^{2}-4 T_{n}\left(T_{n+1}-1\right)}, \\
& \Delta=\frac{-b \pm \sqrt{b^{2}-4 a c}}{2 a},
\end{aligned}
$$

$$
\begin{aligned}
& \delta_{1}=\left(T_{1}-T_{n}-T_{n-1}\right)\left(T_{1}-T_{n+1}\right)^{n-3} \\
& \quad+\sum_{i=2}^{n-2}(-1)^{1+i}\left(T_{1}-T_{n+1}\right)^{n-i-2} \cdot T_{n-i-1} \operatorname{det} A_{i-1}, \\
& \delta_{2}=\sum_{i=1}^{n-2}(-1)^{1+i}\left(T_{1}-T_{n+1}\right)^{n-i-2}\left(T_{n-i+1}-T_{n-i}\right) \operatorname{det} A_{i-1}, \\
& \operatorname{det} A_{i-1} \\
& =\left\{\begin{array}{l}
\frac{\left(\left(b+\sqrt{b^{2}-4 a c}\right) / 2\right)^{i}-\left(\left(b-\sqrt{b^{2}-4 a c}\right) / 2\right)^{i}}{\sqrt{b^{2}-4 a c}}, \\
i\left(\frac{b}{2}\right)^{i-1}, \quad b^{2}=4 a c .
\end{array}\right. \\
& a=T_{1}-T_{n+1}, \quad b=-T_{n}-T_{n-1}, \quad c=-T_{n} .
\end{aligned}
$$

In addition, we will develop solving the problem in [2426] by circulant matrices technology.

\section{Conflict of Interests}

The authors declare that there is no conflict of interests regarding the publication of this paper.

\section{Acknowledgments}

The research was supported by the Development Project of Science \& Technology of Shandong Province (Grant no. 2012GGX10115) and the AMEP of Linyi University, China.

\section{References}

[1] H. Eghbali, S. Muhaidat, S. A. Hejazi, and Y. Ding, "Relay selection strategies for single-carrier frequency-domain equalization multi-relay cooperative networks," IEEE Transactions on Wireless Communications, vol. 12, no. 5, pp. 2034-2045, 2013.

[2] N. Sun and J. Wu, "Optimum sampling in spatial-temporally correlated wireless sensor networks," EURASIP Journal on Wireless Communications and Networking, vol. 2013, article 5, 2013.

[3] S. Arimoto, "Repeat space theory applied to carbon nanotubes and related molecular networks. I," Journal of Mathematical Chemistry, vol. 41, no. 3, pp. 231-269, 2007.

[4] R. H. Chan and W. K. Ching, "Circulant preconditioners for stochastic automata networks," Numerische Mathematik, vol. 87, no. 1, pp. 35-57, 2000.

[5] P. F. Stadler, W. Schnabl, C. V. Forst, and P. Schuster, "Dynamics of small autocatalytic reaction networks-II. Replication, mutation and catalysis," Bulletin of Mathematical Biology, vol. 57, no. 1, pp. 21-61, 1995.

[6] Y. Jing and H. Jafarkhani, "Distributed differential space-time coding for wireless relay networks," IEEE Transactions on Communications, vol. 56, no. 7, pp. 1092-1100, 2008.

[7] G. Q. Wang and S. S. Cheng, "6-periodic travelling waves in an artificial neural network with bang-bang control," Journal of 
Difference Equations and Applications, vol. 18, no. 2, pp. 261-304, 2012.

[8] D. Pais, C. H. Caicedo-Nunez, and N. E. Leonard, "Hopf bifurcations and limit cycles in evolutionary network dynamics," SIAM Journal on Applied Dynamical Systems, vol. 11, no. 4, pp. 1754-1784, 2012.

[9] P. J. Davis, Circulant Matrices, John Wiley \& Sons, New York, NY, USA, 1979.

[10] Z. L. Jiang and Z. X. Zhou, Circulant Matrices, Chengdu Technology University Publishing Company, Chengdu, China, 1999.

[11] C. Erbas and M. M. Tanik, "Generating solutions to the Nqueens problem using 2-circulants," Mathematics Magazine, vol. 68 , no. 5, pp. 343-356, 1995.

[12] Y. K. Wu, R. Z. Jia, and Q. Li, " $g$-circulant solutions to the $(0,1)$ matrix equation $A^{m}=J_{n}^{\star}$, Linear Algebra and Its Applications, vol. 345, no. 1-3, pp. 195-224, 2002.

[13] E. Ngondiep, S. Serra-Capizzano, and D. Sesana, "Spectral features and asymptotic properties for $g$-circulants and $g$ Toeplitz sequences," SIAM Journal on Matrix Analysis and Applications, vol. 31, no. 4, pp. 1663-1687, 2010.

[14] Z. L. Jiang, Y. P. Gong, and Y. Gao, "Circulant type matrices with the sum and product of Fibonacci and Lucas numbers," Abstract and Applied Analysis, vol. 2014, Article ID 375251, 12 pages, 2014.

[15] Z. Jiang, Y. Gong, and Y. Gao, "Invertibility and explicit inverses of circulant-type matrices with $k$-Fibonacci and $k$ Lucas numbers," Abstract and Applied Analysis, vol. 2014, Article ID 238953, 9 pages, 2014.

[16] X. Jiang and K. Hong, "Exact determinants of some special circulant matrices involving four kinds of famous numbers," Abstract and Applied Analysis, vol. 2014, Article ID 273680, 12 pages, 2014.

[17] D. Bozkurt and T. Y. Tam, "Determinants and inverses of circulant matrices with Jacobsthal and Jacobsthal-Lucas Numbers," Applied Mathematics and Computation, vol. 219, no. 2, pp. 544551, 2012.

[18] A. Cambini, "An explicit form of the inverse of a particular circulant matrix," Discrete Mathematics, vol. 48, no. 2-3, pp. 323325, 1984.

[19] S. Q. Shen, J. M. Cen, and Y. Hao, "On the determinants and inverses of circulant matrices with Fibonacci and Lucas numbers," Applied Mathematics and Computation, vol. 217, no. 23, pp. 9790-9797, 2011.

[20] M. Elia, "Derived sequences, the Tribonacci recurrence and cubic forms," The Fibonacci Quarterly, vol. 39, no. 2, pp. 107-115, 2001.

[21] B. Balof, "Restricted tilings and bijections," Journal of Integer Sequences, vol. 15, Article ID 12.2.3, 2012.

[22] S. Rabinowitz, "Algorithmic manipulation of third-order linear recurrences," The Fibonacci Quarterly, vol. 34, no. 5, pp. 447464, 1996.

[23] J. Li, Z. L. Jiang, and F. Lu, "Determinants, norms, and the spread of circulant matrices with Tribonacci and generalized Lucas numbers," Abstract and Applied Analysis, vol. 2014, Article ID 381829, 9 pages, 2014.

[24] D. Ding, Z. Wang, J. Hu, and H. Shu, "Dissipative control for state-saturated discrete time-varying systems with randomly occurring nonlinearities and missing measurements," International Journal of Control, vol. 86, no. 4, pp. 674-688, 2013.

[25] Z. Wang, H. Dong, B. Shen, and H. Gao, "Finite-horizon $H_{\infty}$ filtering with missing measurements and quantization effects,"
IEEE Transactions on Automatic Control, vol. 58, no. 7, pp. 17071718, 2013.

[26] J. Hu, Z. Wang, B. Shen, and H. Gao, "Quantised recursive filtering for a class of nonlinear systems with multiplicative noises and missing measurements," International Journal of Control, vol. 86, no. 4, pp. 650-663, 2013. 


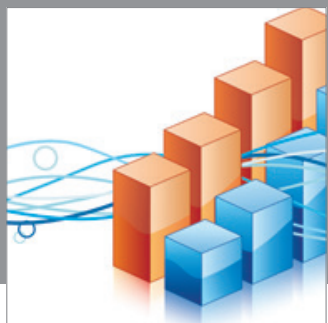

Advances in

Operations Research

mansans

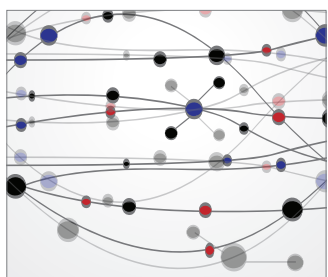

The Scientific World Journal
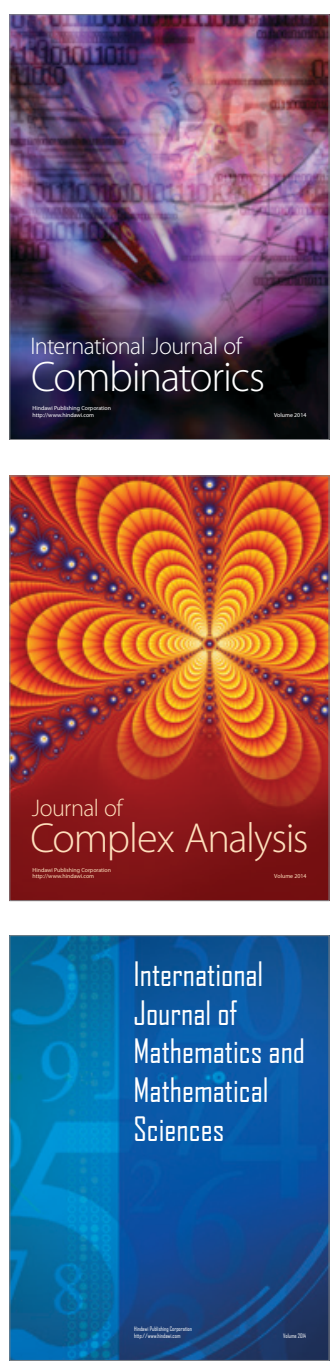
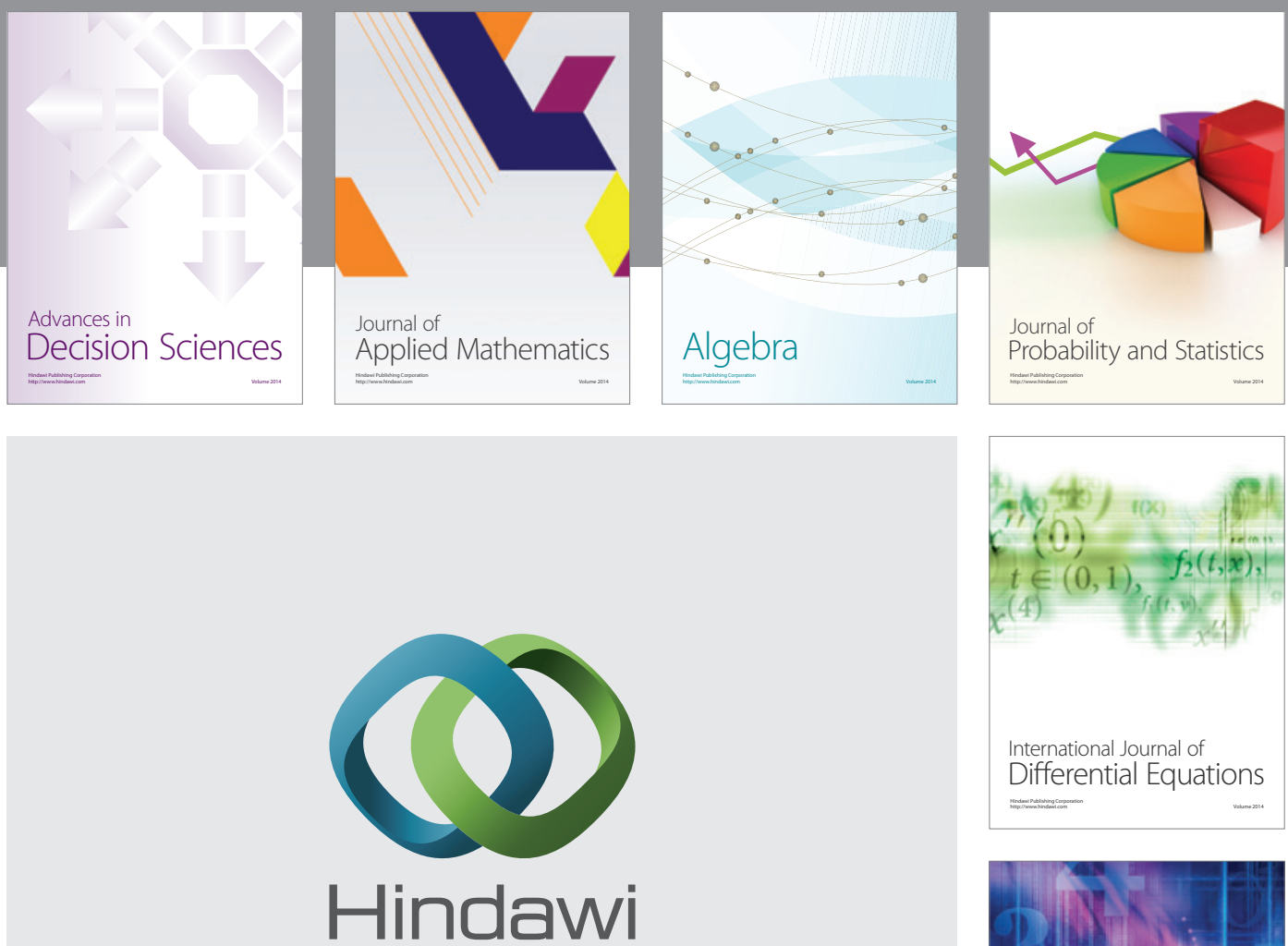

Submit your manuscripts at http://www.hindawi.com
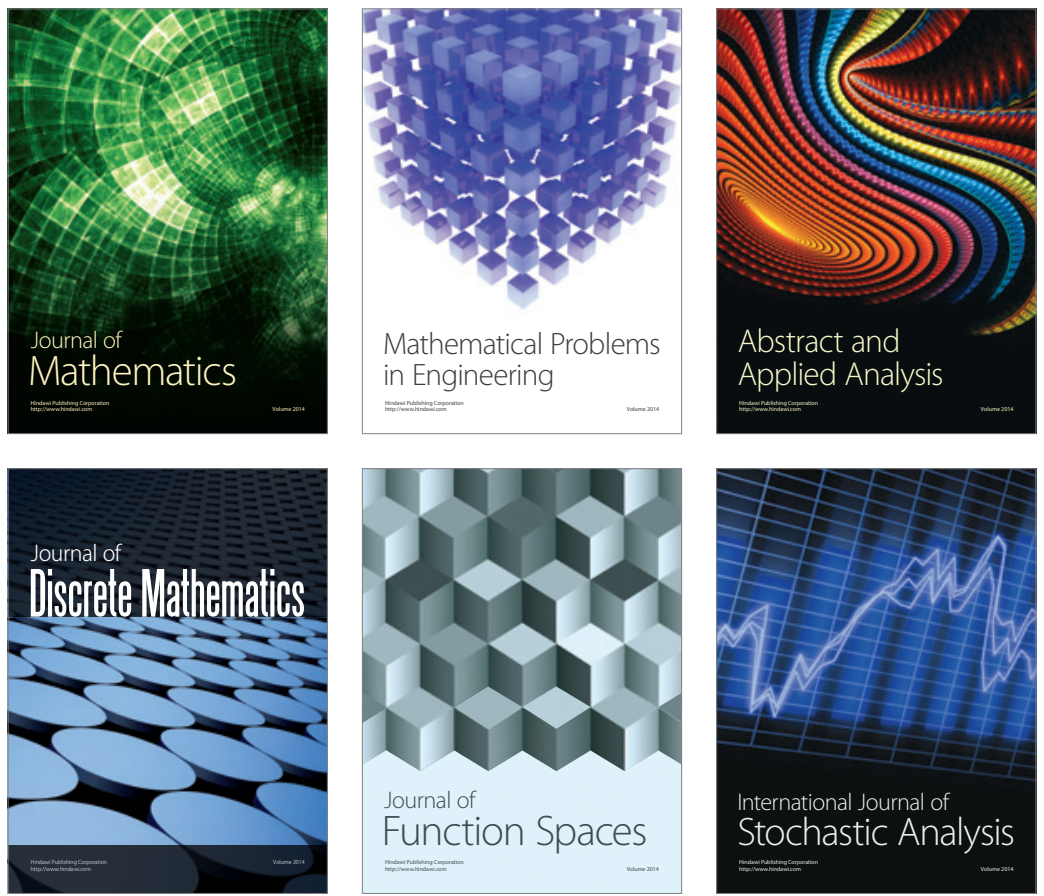

Journal of

Function Spaces

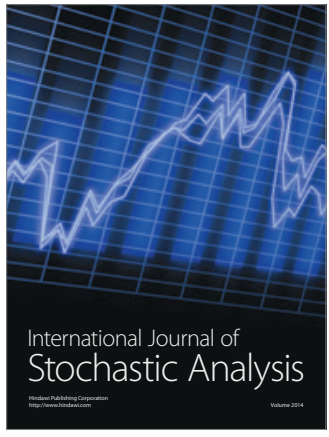

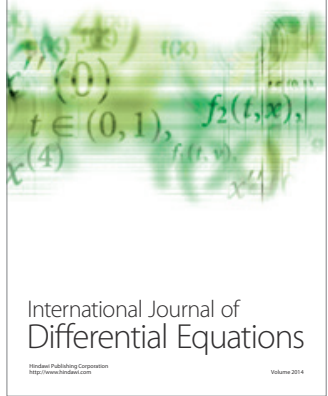
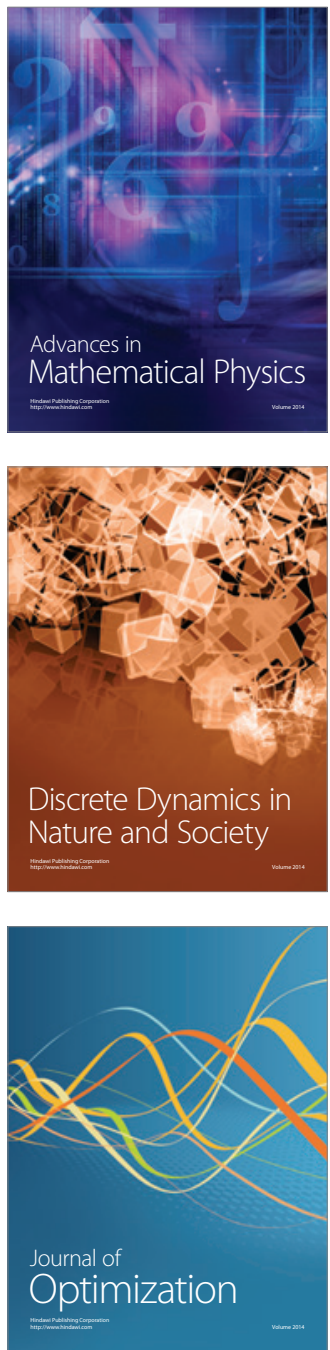